\title{
Enhanced Th17 Responses in Patients with Autoimmune Hepatitis
}

\author{
Farinaz Behfarjam ${ }^{1}$, Siavash Nasseri-Moghaddam ${ }^{2}$, Zohreh Jadali ${ }^{3, *}$
}

1. Ph.D. Student, Clinical Genetics Department, National Institute of Genetic Engineering and Biotechnology Tehran, Iran

2. Associate Professor, Digestive Diseases Research Center, Digestive Diseases Research Institute, Tehran University of Medical Sciences, Tehran, Iran

3. Ph.D., Department of Immunology, School of Public Health, Tehran University of Medical Sciences, Tehran, Iran

\section{* Corresponding Author:}

Zohreh Jadali, Ph.D

Department of Immunology, School of Public Health, Tehran University of Medical Sciences, P.O.BOX: 6446 Tehran 14155, Iran

Tel: + 982166462268

Fax: + 982166462267

Email: zjadali@razi.tums.ac.ir

Received: 18 Nov. 2018

Accepted: 10 Mar. 2019

\section{ABSTRACT}

\section{BACKGROUND}

$\mathrm{T}$ cells are major players in chronic inflammatory diseases such as autoimmune hepatitis (AIH). However, it is not clear which subset of T cells participates in the pathophysiology of the disease. The aim of this study was to assess the expression profile of signature transcription factor and cytokines of T helper 17 (Th17) cells in patients with AIH.

\section{METHODS}

A total of 24 patients with AIH and 24 normal subjects were recruited in the study. Comparison of gene expression patterns between the patients and normal subjects was done by quantitative real-time reverse transcriptase polymerase chain reaction (qRT-PCR).

\section{RESULTS}

The results showed that retinoic acid receptor-related orphan receptors gamma (RORyt), interleukin-17A (IL-17A), and interleukin-22 (IL-22) mRNA expression were increased greatly in the patients group compared with the normal controls group $(p<0.05)$.

\section{CONCLUSION}

Deregulated production of Th17-related molecules may be associated with the pathogenesis of AIH.

\section{KEYWORDS:}

Autoimmune hepatitis, Autoimmunity-Gene Expression Profiling, Cytokines

\section{Please cite this paper as:}

Behfarjam F, Nasseri-Moghaddam S, Jadali Z. Enhanced Th17 Responses in Patients with Autoimmune Hepatitis. Middle East J Dig Dis 2019;11:98-103. doi: 10.15171/mejdd.2018.134.

\section{INTRODUCTION}

Autoimmune hepatitis (AIH) is an organ-specific autoimmune disorder, which tends to affect women more than men. The exact cause of this complex liver disease remains unclear but a combination of genetic and environmental factors is believed to play an important role in the initiation or progression of the disease. ${ }^{1}$

Abnormal immune response is distinguished by qualitative and quantitative alterations of cellular components of the immune system and their products. For instance, increased levels of autoantibodies (the predominant components of humoral immunity) are the hallmark of AIH. Some of these antibodies are directed against liver tissue antigens, including anti-liver kidney microsome ${ }^{1}$, and their titer are associated with disease activity. ${ }^{2}$ Nonetheless, decades of research reveal the importance of $\mathrm{CD}^{+} \mathrm{T}$ helper (Th) cells in disease pathogenesis because they are central to both humoral and cellular immunity. 
Table 1: Primer sequences for the real-time quantitative polymerase chain reaction

\begin{tabular}{ll}
\hline \multirow{2}{*}{ RORyt } & F: 5'AGGAAGTCCATGTGGGAG3' \\
& R: 5'AGCACAATCTGGTCATTCTGG3' \\
\hline \multirow{2}{*}{ IL-17 } & F: 5'CTCATTGGTGTCACTGCT3' \\
& R: 5'CGGTTGTAGTAATCTGAGG3' \\
\hline \multirow{2}{*}{ IL-22 } & F: 5'CAACAGGCTAAGCACATGTCATATTG 3' \\
& R: 5'CAGGCATTTCTCAGAGACATAAACAG3' \\
\hline \multirow{2}{*}{-actin } & F: 5'AGACGCAGGATGGCATGGG3' \\
& R: 5'GAGACCTTCAACACCCCAGCC3' \\
\hline
\end{tabular}

F, forward primer; R, reverse primer; RORyt, retinoic acid receptor-related orphan receptors gamma; IL-17A, interleukin-17A; IL-22, interleukin-22

Recognition and activation of the host Th cells is a crucial step toward tolerance loss and induction of autoimmunity. The immunological microenvironment and nature of antigenic determinants direct the differentiation of naïve $\mathrm{T}$ cells into multiple subpopulations with distinct functional properties. ${ }^{3}$ The specificities of these cells are determined by the expression pattern of the particular cell surface markers, their effector molecules, and cell type specific transcription factors, which are required for controlling $\mathrm{T}$ cell fate decisions. ${ }^{4}$

In recent years, a distinct subset of T helper cells, named Th17 cells, has been described. They constitute a minor functional T-cell population in the peripheral blood (1\%) and should be viewed as a separate lineage because they have a specific gene signature and functions. Th17 cells can be identified by the secretion of specific cytokines, particularly IL-17A and IL-22, and retinoic acid receptorrelated orphan receptors gamma (RORyt) is the critical transcription factor that promotes their differentiation. ${ }^{5}$

Despite the strong body of evidence pointing to the important role of Th17 cells in the pathogenesis of $\mathrm{AIH}$, there are still conflicting reports in the literature..$^{6-8}$ Thus, the objective of this study was firstly to evaluate the pattern of Th17-associated transcription factor RORyt and cytokines (IL-17A, and IL-22) in the peripheral blood mononuclear cells (PBMCs) of patients with AIH and healthy controls, and secondly to examine the relationships between variables measured in this study.

\section{MATERIALS AND METHODS}

\section{Subjects}

A total of 48 participants who comprised 24 patients with AIH and 24 controls were included in this study. Blood samples were taken from all newly diagnosed patients with AIH who were admitted to the outpatient clinic of the Digestive Disease Research Center (DDRC) during the period of the study (2015-17). They had not received prior treatment for $\mathrm{AIH}$ and were matched against the exclusion and inclusion criteria.

Patients were included in the study if they had diagnostic criteria defined by International Autoimmune Hepatitis Group Report. ${ }^{9}$ They also gave informed consent regarding the research. Ethics committee approval was received for this study from the Ethics Committee of Tehran University of Medical Sciences.

Major exclusion criteria were as follows: use of hepatotoxic drugs or herbal medications, treatment with immunosuppressive drugs, and previous diagnosis of chronic inflammatory and autoimmune diseases except AIH. The group of patients consisted of 24 participants who aged between 20 to 69 years.

The control group comprised 24 unrelated, age- and sex-matched subjects who were randomly selected from healthy volunteers. They had no history of chronic inflammatory autoimmune or infectious diseases. The study was conducted according to the ethical principles stated in the Declaration of Helsinki.

\section{Purification of total RNA from PBMCs}

Blood specimens were obtained in 6-mL tubes containing EDTA as anticoagulant. PBMCs were isolated from the whole blood by Ficoll-Paque gradient centrifugation (Pharmacia, Uppsala, Sweden). Total RNA was extracted from PBMCs using RibospinTM (GeneALL, Seoul, Korea) according to the manufacturer's instructions. The RNA quantity and quality was evaluated by Nanodrop spectrophotometer (Thermo Scientific, USA) and agarose gel electrophoresis, respectively.

cDNA synthesis and quantitative real-time reverse transcriptase polymerase chain reaction (qRT-PCR) assay

RNA samples were converted to cDNA using the first strand cDNA synthesis kit (Fermentas, Germany). Amplification and detection were performed with a Rotor-Gene 6000 instrument (Corbett Life Science, Australia), using SYBR Premix EX Taq II kit (Takara, Japan). Primers were designed using Oligo software (National Biosciences) and purchased from Gene fanavaran (Tehran, Iran). PCR primer sequences are indicated in table 1 . The expression 
Table 2: Demographic features of the patients with autoimmune hepatitis and controls according to sex and age

\begin{tabular}{lcccc}
\hline Topics & & Patients N(\%) & Controls N(\%) & \multicolumn{2}{c}{ Statistical significance } \\
\hline Sex & Female & $20(83)$ & $18(75)$ & NS \\
\cline { 2 - 5 } & Male & $4(17)$ & $6(25)$ & NS \\
\cline { 2 - 5 } & Total & $24(100)$ & $24(100)$ & NS \\
\hline Age (years) & Female & $42.90 \pm 12.56$ & $38.56 \pm 14.90$ & NS \\
\cline { 2 - 5 } & Male & $38.50 \pm 14.48$ & $39.13 \pm 11.85$ & NS \\
\cline { 2 - 5 } & Total & $42.17 \pm 12.67$ & $38.75 \pm 13.70$ & NS \\
\hline
\end{tabular}

$\mathrm{N}$ : number of individuals; NS: not significant

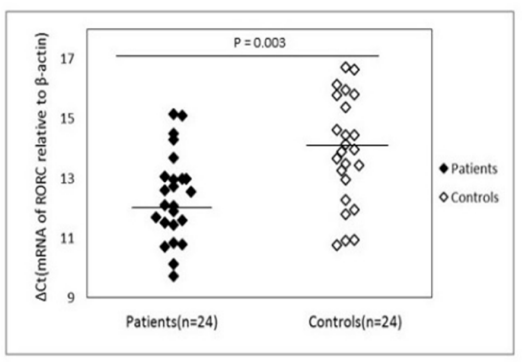

Fig.1: mRNA expression levels for RORC in patients and control subjects. $\beta$-actin was used as the internal control. Please note that a higher $\Delta \mathrm{Ct}$ value corresponds to a comparably lower expression level.

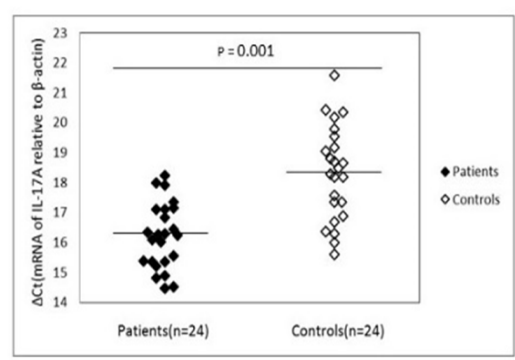

Fig.2: mRNA expression levels for Il-17A in patients and control subjects. $\beta$-actin was used as the internal control. Please note that a higher $\Delta \mathrm{Ct}$ value corresponds to a comparably lower expression level.

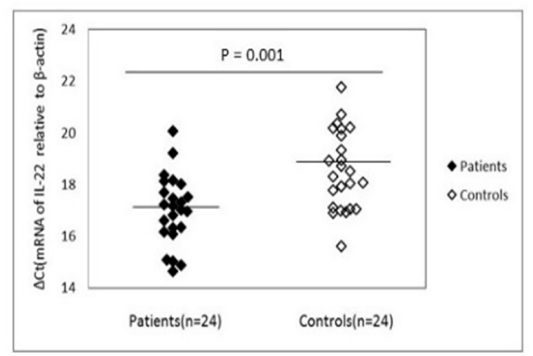

Fig.3: mRNA expression levels for Il-22 in patients and control subjects. $\beta$-actin was used as the internal control. Please note that a higher $\Delta \mathrm{Ct}$ value corresponds to a comparably lower expression level. of constitutively expressed housekeeping gene, $\beta$-actin, was used for normalization.

PCR amplifications were performed in a total volume of $10 \mu \mathrm{L}$, consisting of $5 \mu \mathrm{L}$ SYBR premix, $1 \mu \mathrm{L}$ cDNA, $0.5 \mu \mathrm{L}$ of forward and reverse primers, and $3 \mu \mathrm{L}$ double distilled water.

Cycling conditions were as follows: $95^{\circ} \mathrm{C}$ for 2 minutes followed by $38-45$ cycles of $5 \mathrm{~s}$ at $95^{\circ} \mathrm{C}$ and final step of $20 \mathrm{~s}$ at $62^{\circ} \mathrm{C}$ for RORyt, $45 \mathrm{~s}$ at $57^{\circ} \mathrm{C}$ for IL-17A, $45 \mathrm{~s}$ at $60^{\circ} \mathrm{C}$ for IL-22, and $20 \mathrm{~s}$ at $63^{\circ} \mathrm{C}$ for $\beta$-actin. Relative expression values were then computed by subtracting the threshold cycle $(\mathrm{Ct})$ value of reference gene ( $\beta$-actin) from the $\mathrm{Ct}$ value of a target gene $(\Delta \mathrm{Ct})$.

\section{Statistical Analysis}

Data analysis was done using SPSS software, version 11.0 (SPSS, Inc., Chicago, IL, USA). Statistical comparisons were performed by t test or Pearson test. Difference with a $P$ value less than 0.05 was considered as statistically significant. Results are expressed as means \pm SD.

\section{RESULTS}

\section{Expression of Th17-related genes in patients with AIH}

Table 2 summarizes the obtained demographic information of the participants. Gene expression levels were measured in PBMCs from patients with AIH and healthy controls. The comparison of mRNA expression of Th17related genes between the patients and control groups are shown in figures 1-3. There was a significant increase in mRNA expression of RORyt between the patients (12.39 $\pm 1.47)$ and controls $(13.90 \pm 1.81)(p=0.003)$.

Compared with healthy individuals, the expression of IL-17A mRNA exhibited a statistically significant increase $(p=0.001)$ in patients' PBMCs $(16.21 \pm 1.10)$ than in healthy controls $(18.31 \pm 1.56)$. Consistently, the expression of pro-inflammatory cytokines IL-22 (figure $3)$, was higher $(p=0.001)$ in patients $(17.01 \pm 1.34)$ than in normal controls $(18.57 \pm 1.53)$.

\section{Correlation analysis}

The mRNA expression of RORyt was correlated positively with mRNA expression of IL-17A $(r=0.419$, 
$p=0.041)$. Statistical analysis indicated that in patients, IL-17A mRNA level was significantly correlated with IL-22 mRNA level ( $\mathrm{r}=0.572, p=0.003)$. However, expression profiles were not affected by sex, age, and liver enzyme tests.

\section{DISCUSSION}

Th17 cells represent an important subset of $\mathrm{CD}^{+} \mathrm{T}$ cells, which are involved in protective immune response to infectious agents, particularly, extracellular pathogens. Nonetheless, uncontrolled or deranged Th17 cells activities can influence the risk for development of autoimmune disorders. ${ }^{10}$ Therefore, investigation of possible changes in Th17-related molecules provides new hints to the molecular mechanisms of the underlying autoimmunity.

Recent studies have established a complex relationship between Th17 cells and other types of effector cells especially Th1 cells. They may act differentially or in collaboration with other immune cells and any disturbances that affect the balance between these cells and their interactions may have a negative impact on the immune system and may enhance vulnerability to a range of different autoimmune diseases. ${ }^{11}$

Th17 cells can be identified by the secretion of important effector cytokines including IL-17A and IL-22 and the transcription factor RORyt is essential for the generation of this lineage. ${ }^{12}$ Moreover, a dysregulated Th17 cell activity, has been linked to different types of autoimmune disorders. ${ }^{10}$ Therefore, quantitative analysis of Th17-related gene expression patterns can be used to elucidate the possible immunopathological mechanisms involved in AIH.

The first step of the present study was to determine the expression pattern of RORyt in PBMCs of patients with AIH. The results revealed increased transcript levels of ROR $\gamma t$ in patients compared with normal controls. To our knowledge, this is the first report for expression analysis of ROR $\gamma t$ in PBMCs of patients with AIH. However, according to a recent report by Mitra and colleagues, ${ }^{13}$ ROR $\gamma t$ mRNA was significantly upregulated in whole blood cells of patients with active disease compared with FOXP3 as a lineage-specific marker of regulatory $\mathrm{T}$ (Treg) cells.

This observation is also consistent with the results, indicating higher percentage of IL-17-producing and
RORC + cells within a fraction of $\mathrm{CD}^{+} \mathrm{T}$ cells that expresses Tim3 (a receptor on $\mathrm{CD}^{+}$effector cells). ${ }^{14}$ Therefore, it is tempting to speculate that RORyt may play a role in the pathogenesis of AIH.

In the next step, the expression pattern of IL-17A and IL-22 was determined in the PBMCs of the participants. The results showed that mRNA levels of IL-17A and IL-22 were significantly increased in PBMCs of patients with AIH compared with normal controls.

These results support earlier evidence from both experimental models and clinical studies that indicate increased expression of IL-17A and IL-22 transcripts in patients with AIH. Researches have also revealed a positive correlation between the levels of the above mentioned cytokines and severity of disease. .,15,16 $^{6}$

Nonetheless, the exact role of these cytokines in the pathogenesis of AIH is not well established yet. For instance, the results of murine experimental model of concanavalin A (ConA)-induced AIH have shown that IL-17-deficient mice develop the same level of liver injury as wild-type mice. ${ }^{17}$ In contrast, the results from two independent research studies indicated that IL-17-deficient mice had significant reduction in liver injury compared with wild-type mice. ${ }^{18,19}$

Therefore, more attention must be paid to clarify the actual cause of IL-17 alterations and its role in vulnerability or resistance to disease process. It seems that IL-17 acts as a double-edged sword in AIH and has complex characteristics in disease pathogenesis. This issue is important because IL-17 receptors are expressed ubiquitously on all types of liver cells (such as hepatocytes, Kupffer cells, stellate cells, and biliary epithelial cells). ${ }^{20}$

The experimental results show that interactions between IL-17 and its receptor induce the expression of multiple factors including acute phase C-reactive protein ${ }^{21}$ and several proinflammatory cytokines/chemokines in liver cells, which may participate in liver inflammation. ${ }^{22}$ In contrast, there are reports indicating that IL-17 has also the ability to ameliorate hepatocytes apoptosis. ${ }^{19}$ Therefore, further studies are needed to fully elucidate the precise role of IL-17 in AIH.

Controversy also exists concerning the role of IL-22 in the AIH. This product of immune cells can affect many parts of the body including the liver. ${ }^{23}$ Similar to IL-17, 
IL-22 is a dual-function cytokine, which may act as a hepatoprotective agent or may contribute to liver problems. The protective activities of IL-22 on the liver are indicated by different studies. For instance, Zenewicz and colleagues demonstrated that in vivo IL-22 could protect hepatocytes against the damaging effects of immune cells in the inflamed liver. ${ }^{17}$ This hepatoprotective effect of IL-22 has also been reported in an experimental model of T-cell mediated hepatitis. ${ }^{24}$

These findings are not consistent with other studies that show the participation of IL-22 in liver damage. For instance, decreased liver inflammation and fibrosis progression was indicated following IL-22 blockade. ${ }^{25}$ Moreover, a positive correlation was observed between the severity of liver disease in patients with cirrhosis or hepatitis B virus (HBV) and IL-22 level. ${ }^{25-27}$

These opposing effects of IL-22 may depend on different factors such as location of action, receptor expression pattern, time of exposure, and dose.

The existence of different cellular sources of these cytokines and the responding cell types are other factors that complicate identification of the exact function of this cytokine in the pathophysiological processes of liver injury.

Another important challenge arises as a result of a complex interaction between different immune cell subtypes and their products. Therefore, it is difficult to estimate the role that specific immune cells play in AIH.

This interpretation is supported by findings from previous studies that indicated increased mRNA expression of Th1-related transcription factor (T-bet) and cytokine (interferon- $\gamma$ ) in patients with $\mathrm{AIH} .{ }^{28}$ These findings add to a growing body of literature on the importance of Th17 and Th1 cells responses in the development of human autoimmunity. ${ }^{29,30}$

This study has some limitations that need to be discussed. The first is the lack of quantitative data concerning the expression of Th17-related genes within the liver of the patients with AIH. The second is the absence of any experimental design for quantitative evaluation of the treatment-related changes in RORyt, IL-17A, and IL-22 mRNA expression levels. Comparison of the gene expression profiles before and after treatment will certainly improve our understanding of the molecular mechanisms of the disease process. Therefore, more studies are needed to determine the clinical importance of Th17 cells in the pathogenesis of AIH.

In conclusion, the present study indicates a marked increased expression of key transcription factor and cytokines for Th17 cells in PBMCs of patients with AIH. These data provide additional information about the potential role of Th17 cells in the pathogenesis of this important liver disease. However, further studies are required to explain different factors that affect Th17 responses. These findings also offer the promise of novel therapies through modulation of $\mathrm{T}$ cell products and functions.

\section{ETHICAL APPROVAL}

There is nothing to be declared.

\section{CONFLICT OF INTEREST}

The authors declare no conflict of interest related to this work.

\section{REFERENCES}

1. Aizawa Y, Hokari A. Autoimmune hepatitis: current challenges and future prospects. Clin Exp Gastroenterol 2017;10:9-18. doi:10.2147/CEG.S101440.

2. Gregorio GV, McFarlane B, Bracken P, Vergani D, Mieli-Vergani G. Organ and non-organ specific autoantibody titres and $\operatorname{IgG}$ levels as markers of disease activity: a longitudinal study in childhood autoimmune liver disease. Autoimmunity 2002;35:515-9. doi: 10.1080/0891693021000056721.

3. Liberal R, Krawitt EL, Vierling JM, Manns MP, MieliVergani G, Vergani D. Cutting edge issues in autoimmune hepatitis. J Autoimmun 2016;75:6-19. doi:10.1016/j. jaut.2016.07.005.

4. Hirahara K, Nakayama T. CD4+ T-cell subsets in inflammatory diseases: beyond the Th1/Th2 paradigm. Int Immunol 2016;28:163-71. doi:10.1093/intimm/dxw006.

5. Eyerich S, Zielinski CE. Defining Th-cell subsets in a classical and tissue-specific manner: Examples from the skin. Eur J Immunol 2014;44:3475-83. doi:10.1002/eji.201444891.

6. Zhao L, Tang Y, You Z, Wang Q, Liang S, Han X, et al. Interleukin-17 contributes to the pathogenesis of autoimmune hepatitis through inducing hepatic interleukin-6 expression. PLoS One 2011;6:e18909. doi:10.1371/journal. pone. 0018909 .

7. Thomas-Dupont P, Remes-Troche JM, Izaguirre-Hernández IY, Sánchez-Vargas LA, Maldonado-Rentería Mde J, Hernández-Flores KG, et al. Elevated circulating levels of 
IL-21 and IL-22 define a cytokine signature profile in type 2 autoimmune hepatitis patients. Ann Hepatol 2016;15:550-8.

8. Liberal R, Grant CR, Mieli-Vergani G, Vergani D, Longhi MS. PWE-281 Different effector T cell responses may account for different patterns of liver injury in childhood autoimmune liver disease. Gut 2012;61(Suppl. 2):A412. doi:10.1136/gutjnl-2012-302514d.281

9. Alvarez F, Berg PA, Bianchi FB, Bianchi L, Burroughs AK, Cancado EL, et al. International Autoimmune Hepatitis Group Report: review of criteria for diagnosis of autoimmune hepatitis. J Hepatol 1999;31:929-38. doi: 10.1016/S0168-8278(99)80297-9.

10. Hoe E, Anderson J, Nathanielsz J, Toh ZQ, Marimla $\mathrm{R}$, Balloch A, et al. The contrasting roles of Th17 immunity in human health and disease. Microbiol Immunol 2017;61:49-56. doi:10.1111/1348-0421.12471.

11. Damsker JM, Hansen AM, Caspi RR. Th1 and Th17 cells: adversaries and collaborators. Ann N Y Acad Sci 2010;1183:211-21. doi:10.1111/j.1749-6632.2009.05133.x.

12. Kuwabara T, Ishikawa F, Kondo M, Kakiuchi T. The role of IL-17 and related cytokines in inflammatory autoimmune diseases. Mediators Inflamm 2017;2017:3908061. doi:10.1155/2017/3908061.

13. Mitra S, Anand S, Das A, Thapa B, Chawla YK, Minz RW. A molecular marker of disease activity in autoimmune liver diseases with histopathological correlation; FoXp3/ROR $\gamma \mathrm{t}$ ratio. APMIS 2015;123:935-44. doi: 10.1111/apm.12457.

14. Liberal R, Grant CR, Holder BS, Ma Y, Mieli-Vergani G, Vergani $\mathrm{D}$, et al. The impaired immune regulation of autoimmune hepatitis is linked to a defective galectin-9/tim-3 pathway. Hepatology 2012;56:677-86. doi: 10.1002/hep.25682.

15. Yan S, Wang L, Liu N, Wang Y, Chu Y. Critical role of interleukin-17/ interleukin-17 receptor axis in mediating Con A-induced hepatitis. Immunol Cell Biol 2012;90:4218. doi:10.1038/icb.2011.59.

16. Dambacher J, Beigel F, Zitzmann K, Heeg MH, Göke B, Diepolder HM, et al. The role of interleukin-22 in hepatitis C virus infection. Cytokine 2008;41:209-16. doi: 10.1016/j.cyto.2007.11.016.

17. Zenewicz LA, Yancopoulos GD, Valenzuela DM, Murphy AJ, Karow M, Flavell RA. Interleukin-22 but not interleukin-17 provides protection to hepatocytes during acute liver inflammation. Immunity 2007;27:647-59. doi: 10.1016/j.immuni.2007.07.023

18. Nagata T, McKinley L, Peschon JJ, Alcorn JF, Aujla SJ, Kolls JK. Requirement of IL-17RA in Con A induced hepatitis and negative regulation of IL-17 production in mouse T cells. J Immunol 2008;181:7473-9. doi:10.4049/ jimmunol.181.11.7473.

19. Lafdil F, Wang H, Park O, Zhang W, Moritoki Y, Yin S, et al. Myeloid STAT3 inhibits T cell-mediated hepatitis by regulating T helper 1 cytokine and interleukin-17 production. Gastroenterology 2009;137:2125-35. doi:10.1053/j. gastro.2009.08.004.

20. Meng F, Wang K, Aoyama T, Grivennikov SI, Paik Y, Scholten D, et al. Interleukin-17 signaling in inflammatory, Kupffer cells, and hepatic stellate cells exacerbates liver fibrosis in mice. Gastroenterology 2012;143:76576. doi:10.1053/j.gastro.2012.05.049.

21. Patel DN, King CA, Bailey SR, Holt JW, Venkatachalam $\mathrm{K}$, Agrawal A, et al. Interleukin-17 stimulates C-reactive protein expression in hepatocytes and smooth muscle cells via p38 MAPK and ERK1/2-dependent NF-kappaB and C/EBPbeta activation. J Biol Chem 2007;282:2722938. doi:10.1074/jbc.M703250200.

22. Lafdil F, Miller AM, Ki SH, Gao B. Th17 cells and their associated cytokines in liver diseases. Cell Mol Immunol 2010;7:250-4. doi:10.1038/cmi.2010.5.

23. Khawar MB, Azam F, Sheikh N, Abdul Mujeeb K. How Does Interleukin-22 Mediate Liver Regeneration and Prevent Injury and Fibrosis? J Immunol Res 2016;2016:2148129. doi:10.1155/2016/2148129.

24. Radaeva S, Sun R, Pan HN, Hong F, Gao B. Interleukin 22 (IL-22) plays a protective role in T cell-mediated murine hepatitis: IL-22 is a survival factor for hepatocytes via STAT3 activation. Hepatology 2004;39:1332-42. doi: 10.1002/hep.20184.

25. Zhao J, Zhang Z, Luan Y, Zou Z, Sun Y, Li Y, et al. Pathological functions of interleukin-22 in chronic liver inflammation and fibrosis with hepatitis B virus infection by promoting $\mathrm{T}$ helper 17 cell recruitment. Hepatology 2014;59:1331-42. doi:10.1002/hep.26916.

26. Zhang Y, Cobleigh MA, Lian JQ, Huang CX, Booth CJ, Bai XF, et al. A proinflammatory role for interleukin-22 in the immune response to hepatitis B virus. Gastroenterology 2011;141:1897-906. doi:10.1053/j.gastro. 2011.06.051.

27. Kronenberger B, Rudloff I, Bachmann M, Brunner F, Kapper L, Filmann N, et al. Interleukin-22 predicts severity and death in advanced liver cirrhosis: a prospective cohort study. BMC Med 2012;10:102. doi:10.1186/1741-7015-10-102.

28. Behfarjam F, Sanati MH, Nasseri Moghaddam S, Ataei M, Nikfam S, Jadali Z. Role of Th1/Th2 cells and related cytokines in autoimmune hepatitis. Turk $J$ Gastroenterol 2017;28:110-4. doi:10.5152/tjg. 2017. 17501.

29. Diani M, Altomare G, Reali E. T Helper Cell Subsets in Clinical Manifestations of Psoriasis. J Immunol Res 2016;2016:7692024. doi:10.1155/2016/7692024.

30. Azadegan-Dehkordi F, Bagheri N, Shirzad H, Rafieian-Kopaei M. The role of Th1 and Th17 cells in glomerulonephritis. J Nephropathol 2015;4:32-7. doi:10.12860/jnp.2015.07. 